\title{
Skeleton Composition using Remote Data
}

\author{
Mischa Dieterle, Thomas Horstmeyer, and Rita Loogen \\ Philipps-Universität Marburg, Fachbereich Mathematik und Informatik \\ Hans Meerwein Straße, D-35032 Marburg, Germany \\ \{dieterle, horstmey, loogen\} einformatik.uni-marburg.de
}

\begin{abstract}
Skeletons simplify parallel programming by providing general patterns of parallel computations. When several skeletons are used inside the same program, skeleton composition usually leads to aggregation and redistribution of the intermediate data on a single process. Though the programmer can overcome the performance loss at a lower level of abstraction by altering the existing skeletons or not using them at all. A high-level concept like skeleton-based programming, however, calls for a more general solution.

Remote data provides runtime mechanisms that allow declaratively specified processes to access other processes' data via remote handles. This enables the programmer to easily build complex skeletons by combining simpler ones. Skeletons can be composed without the drawback of collecting and then redistributing the data in between two skeleton instances. Another advantage is that skeletons which inherently depend on their inner communication patterns are easily implemented using remote data. We present the implementation of remote data in the parallel functional language Eden and show the definition of some example skeletons with a remote data interface.
\end{abstract}

Keywords: skeletons, composition, parallel, functional

\section{Introduction}

Algorithmic skeletons [5] capture common patterns of parallel evaluations like task farms, pipelines, divide-and-conquer schemes etc. The application programmer only needs to instantiate a skeleton appropriately, thereby concentrating on the problem-specific matters and trusting on the skeleton with respect to all parallel details. Skeletons should be small and simple to instantiate to increase the ease and flexibility of their use. In particular, it should be possible to compose and nest skeleton instantiations arbitrarily. This means for the case of a distributed memory setup and structured data that must be passed from one skeleton to the next that the result of the first skeleton is gathered in a single process and redistributed for the following skeleton execution. This causes unnecessary communication and holds the danger of a communication bottleneck in the caller process (see Fig. 1 (a)). A typical example is the composition of two parallel maps (parallel task farms) producing a two dimensional matrix with an intermediate transpose.

In Manuel Carro and Ricardo Pea, editors, Practical Aspects of Declarative Languages 12th International Symposium, PADL 2010, LNCS 5937, pages 73-87, Madrid, Spain, January 2010. (C) 2010 Springer-Verlag Berlin/Heidelberg

The original publication is available at springerlink.com 
Dieterle, Horstmeyer, Loogen, Skeleton Composition using R. D. In: PADL 2010, LNCS 5937. (C) 2010 Springer Berlin/Heidelberg. The original publication is available at springerlink.com

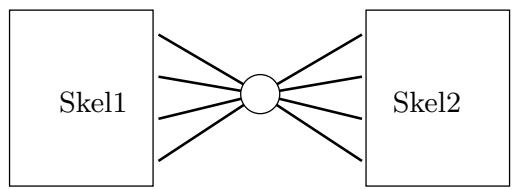

(a) collection and redistribution

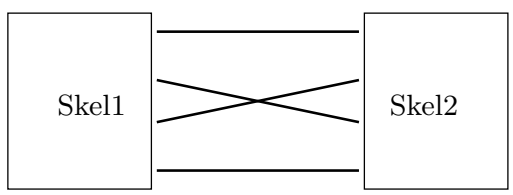

(b) direct redistribution

Fig. 1. Data transfer between composed skeleton instances

There exist several proposals to avoid the gathering and redistribution of distributed data. One could introduce a new distributed data type as common in languages with a data-parallel concept $[7,6]$ where data can be passed in a distributed manner. In this case, one needs special transformation and conversion functions to redistribute the distributed data or to switch between distributed and common data types. Another simple alternative would be to design a new integrated skeleton for the composition by merging the two skeleton instantiations and organising the redistribution explicitly within the new skeleton context. This approach has the disadvantage that the programmer has to go into the internals of skeleton design and that the clarity of the original composition is lost.

In this paper, we present an alternative approach that allows the direct passing of distributed result data from one skeleton instance to the next one (see Fig. 1 (b)). The main idea is to replace the data by handles to it, called remote data, which are gathered and redistributed instead. The handles can then be used to pull the real data directly to the target. This concept which has independently been suggested by Alt and Gorlatch $[2,3,1]$ can be easily used: normal data is replaced by the corresponding remote data handles and skeletons that operate on the new remote data can be composed as before. Only that now the gathering and redistribution of complex data is replaced by the gathering and exchange of small remote data handles which are used for the direct data exchange between processes within different skeleton instances. Thus, remote data handles for data which may be located elsewhere can be used like the original data but cause only low communication costs. They can occur everywhere where ordinary data may occur, e.g. in lists or trees to model distributed data structures. As we will show, this concept is flexible to use and still type-safe.

We develop the concept of remote data in the context of our parallel functional language Eden, although the concept itself is language-independent. It could equally well be added to other parallel languages, see [2, 3, 1] for a realisation in Java. The realisation in a declarative language has the advantage that the beauty and elegance of declarative programming is maintained for parallel skeleton-based programming. In functional languages, skeletons are realised as higher order functions. Skeleton instantiation reduces to function application and skeleton composition is nothing else than function composition.

We will introduce a new data type RD a representing a handle for remote data of type a and provide interface functions release $:: a \rightarrow R D$ a and fetch $:: \mathrm{RD}$ a $\rightarrow$ a. The function release yields a remote data handle that can be passed to other processes, which will in turn use the function fetch 
Dieterle, Horstmeyer, Loogen, Skeleton Composition using R. D. In: PADL 2010, LNCS 5937. (C) 2010 Springer Berlin/Heidelberg. The original publication is available at springerlink.com

to access the remote data. The data transmission occurs automatically from the processes that released the data to the process which uses the handle to fetch the remote data. Skeleton composition skel2 $\circ$ skell of type a $\rightarrow c$ where skel2 of type $\mathrm{b} \rightarrow \mathrm{c}$ and skel1 of type $\mathrm{a} \rightarrow \mathrm{b}$ will now be replaced by skel2' $\circ$ skel1' of type $a \rightarrow c$ where skel2' is of type RD b $\rightarrow c$ and skel1' is of type $a \rightarrow R D$ b. The modified skeleton definitions differ from the original ones only in additional applications of release in skel1' and fetch in skel2'. These small modifications solve our problem while preserving the original program structure. We will show that complex communication structures like an all-to-all scheme can easily and elegantly be defined using remote data.

Plan of the paper In Section 2 we give a introduction to the language Eden. Section 3 presents the implementation of the new data type constructor RD with interface functions fetch and release in Eden. Section 4 shows how to use remote data for skeleton composition and the definition of complex communication patterns. Section 5 compares with related work while Section 6 finally concludes.

\section{Eden in a Nutshell}

The parallel Haskell dialect Eden [9] extends Haskell with an explicit notion of processes (function applications evaluated remotely in parallel). The programmer has direct control over evaluation site, process granularity, data distribution and communication topology, but does not have to manage synchronisation and data exchange between processes. The latter are performed by the parallel runtime system through implicit communication channels, transparent to the programmer.

The essential two coordination constructs of Eden are process abstraction and instantiation:

process : : (Trans a, Trans b) $\Rightarrow(\mathrm{a} \rightarrow \mathrm{b}) \rightarrow$ Process $\mathrm{a}$ b

$(\#) \quad:$ : (Trans $\mathrm{a}$, Trans b) $\Rightarrow$ Process $\mathrm{a} b \rightarrow \mathrm{b} \rightarrow \mathrm{b}$

The function process embeds functions of type $\mathrm{a} \rightarrow \mathrm{b}$ into process abstractions of type Process a b where the context (Trans a, Trans b) states that both $\mathrm{a}$ and $\mathrm{b}$ must be types belonging to the Trans class of transmissible values. Evaluation of an expression (process funct) \# arg leads to the creation of a new process for evaluating the application of the function funct to the argument arg.

For immediately instantiating a list of process abstractions with appropriate inputs, Eden provides a (predefined) function spawn, and a variant spawnAt which additionally locates the created processes on given processor elements. Neglecting demand control, spawn is denotationally specified, and could be defined, by the following equation.

spawn : : (Trans $a$, Trans b) $\Rightarrow$ [Process a b] $\rightarrow[a] \rightarrow[b]$

spawn $=$ zipwith $(\#)$ 
Dieterle, Horstmeyer, Loogen, Skeleton Composition using R. D. In: PADL 2010, LNCS 5937. (C) 2010 Springer Berlin/Heidelberg. The original publication is available at springerlink.com

Eden further provides functions to create and use explicit connections between arbitrary processes.

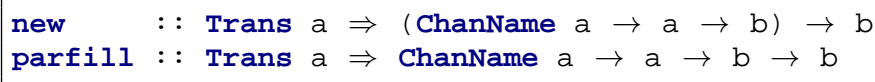

They can be used to shortcut the tree-shaped topologies created by the basic functions. The function new is used at the receiver side to created a receiver port of a unidirectional channel connection. It works in continuation passing style, new's parameter function's first parameter is the "name" of the channel (type ChanName a) whose incoming port is created as a side effect. The second parameter is the value that will be received via the channel. The parameter function's output is the result of the function new. The sender of the connection is still not determined. The channel's "name" can be passed to another process. The connection gets established when parfill is used at the sender side using the "name" of the channel (containing the receivers process ID and port). The function parfill takes the value to be written in the channel, parfill's third argument is returned unchanged after forking a thread that sends the data through the channel.

These two function are quite complicated to use for people new to Eden. Their signatures interplay well in some circumstances, but they are not intuitive at all. The main problem when using dynamic channels is the change of direction in the communication: when Process 1 wants to send data directly to Process 2 using a dynamic channel, this channel must first be generated by Process 2 and sent from Process 2 to Process 1 before the proper data transfer from Process 1 to Process 2 can take place. Thus, the dynamic channel must be communicated in the opposite direction in which the data is to be transferred. This complicates the use of dynamic channels. The remote data approach keeps the direction of the communication by introducing another channel transfer from Process 1 to Process 2. This transfer sends a channel via which Process 2 can send its data channel to Process 1. Thus, an exchange of dynamic channels takes place between Process 1 and Process 2 which automatically establishes a data channel connection from Process 1 to Process 2. In the following, we implement the remote data concept using Eden's dynamic channels. Note that this concept provides the same expressive power as dynamic channels, but in a more natural and easier-to-use way.

\section{Eden Implementation of Remote Data}

The implementation of remote data in Eden (Figure 2) is simple and elegant. To release a local data $\mathrm{x}$ of type a we create - using the function new a channel name cc of type ChanName (ChanName a) via which a channel $c$ of type ChanName a will be received. Using parfill a thread is forked that subsequently sends the local data $x$ via the channel $c$. The result of the release function is the newly created channel cc : : ChanName (ChanName a). Note that the remote data type $\mathrm{RD}$ a is a synonym of CC's type. Data of type RD a 
Dieterle, Horstmeyer, Loogen, Skeleton Composition using R. D. In: PADL 2010, LNCS 5937. (C) 2010 Springer Berlin/Heidelberg. The original publication is available at springerlink.com

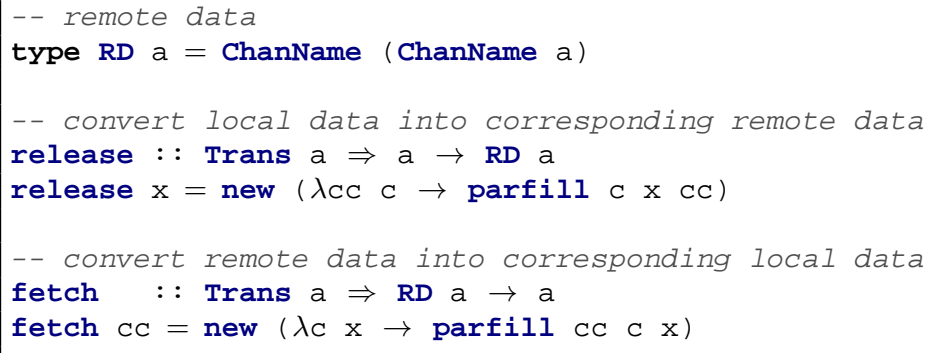

Fig. 2. Remote data definition
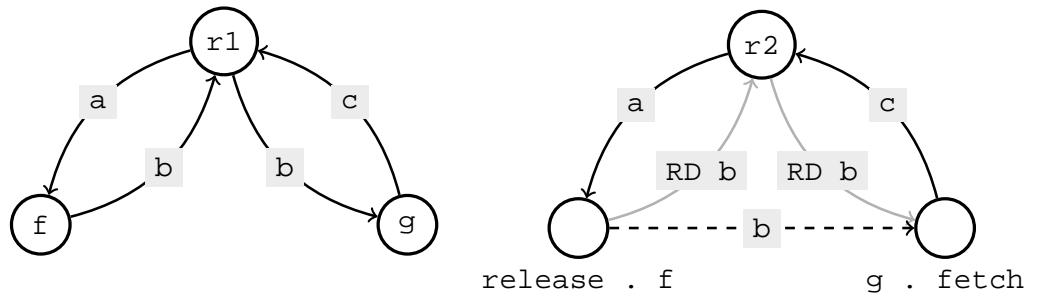

Fig. 3. Using remote data

is merely a channel name and thus very lightweight with low communication costs. To access remote data we need to fetch it by again creating a channel c : : ChanName a using the function new. This channel is sent via the remote data handle, i.e. the channel $\mathrm{Cc}$ of type RD a. The proper data is then received via channel $\mathrm{C}$ and returned as the result of the fetch function.

A problem arises when remote data needs to be duplicated. Channel names (of type ChanName a) cannot be used more than once to retain referential transparency [9]. As remote data is implemented as a specialized channel name, it must not be duplicated and fetched several times in parallel. A manual workaround to duplicate remote data on a node would be to fetch the data and release it again repeatedly. We considered more sophisticated versions which make the use of remote data more comfortable, but they expose nondeterminism and should therefore not be implemented in the actual version of Eden.

Our new way of communication creates a slight overhead. In comparison to the common way of defining explicit communication we have an additional channel per direct connection that is used only before the transmission of the actual data begins. However, as this channel only transports a value of type ChanName a which is quite small the increase in communication cost should not be noticeable in most cases.

Example. We show a small example where the remote data concept is used to establish a direct channel connection between sibling processes. Given functions $\mathrm{f}$ and $\mathrm{g}$, one can calculate $(\mathrm{g} \circ \mathrm{f}$ ) a in parallel creating a process for each 
Dieterle, Horstmeyer, Loogen, Skeleton Composition using R. D. In: PADL 2010, LNCS 5937. (C) 2010 Springer Berlin/Heidelberg. The original publication is available at springerlink.com

function. Figure 3 shows two different ways to implement this. Simply replacing the function calls by process instantiations

$$
r 1 \mathrm{a}=\text { process } \mathrm{g} \# \text { (process } \mathrm{f} \# \mathrm{a} \text { ) }
$$

leads to the following behaviour (visualised in the left part of Fig. 3): Function $r 1$ instantiates the first process calculating $\mathrm{f}$, passes its input to this process and receives the remotely calculated result. It instantiates a second process calculating $g$ and passes the result of process $f$ to this new process. The output of the second process is also sent back to the caller. The drawback of this approach is that the result of the first process will not be sent directly to the second process. This causes unnecessary communication costs.

We use remote data RD a in the second implementation

r2 a process ( $g \circ$ fetch) \# (process (release $\circ$ f) \# a).

It uses function release to produce a handle of type RD a for data of type a. Calling fetch with remote data returns the value released before. Function $r 2$ is identical to $r 1$ except for the conversion of the result type of $f$ 's process and the input type of g's process to remote data. The use of remote data leads to a direct communication of the actual data between the processes of $f$ and $g$ (see the right part of Fig. 3). The remote data handles are treated like the original data in the first version and the basic structure of the program, i.e. the composition of two process instantiations, remains the same.

\section{Composing Predefined Skeletons}

Before handling the composition of skeletons using the remote data concept, we show the lifting of a simple parallel map skeleton to a remote data interface. Then we define a parallel all-to-all skeleton which generates a number of processes each of which exchanges data with any of the others. Using these skeletons with their remote data interfaces enables us to define a sequence consisting of a parallel map, a parallel transpose (realised using the all-to-all skeleton) and a second parallel map. This can be useful in an implementation of a parallel FFT skeleton [8] or a Google Map-Reduce skeleton [4]. In [4, 8], corresponding parallel map-transpose skeletons have been defined as monolithic skeletons without composing simpler skeletons. With the remote data interface, we can define the same skeleton as a composition of the three component skeletons. This leads to a much better understandable definition while achieving the same performance. Finally, we present another elegant and concise definition of an even more complex communication pattern: a butterfly scheme which is used to define an all-reduce-skeleton.

\subsection{The parmapDC skeleton}

A parallel map creates a process for each element of the input list. In Eden, it can easily be defined using the function spawn (see Fig. 4). Note that this definition implies that the process evaluating parmap creates as many processes as there are elements in the input list and sends each of theses elements to the corresponding process. Using a remote data interface, each process only gets a handle to 
Dieterle, Horstmeyer, Loogen, Skeleton Composition using R. D. In: PADL 2010, LNCS 5937. (C) 2010 Springer Berlin/Heidelberg. The original publication is available at springerlink.com

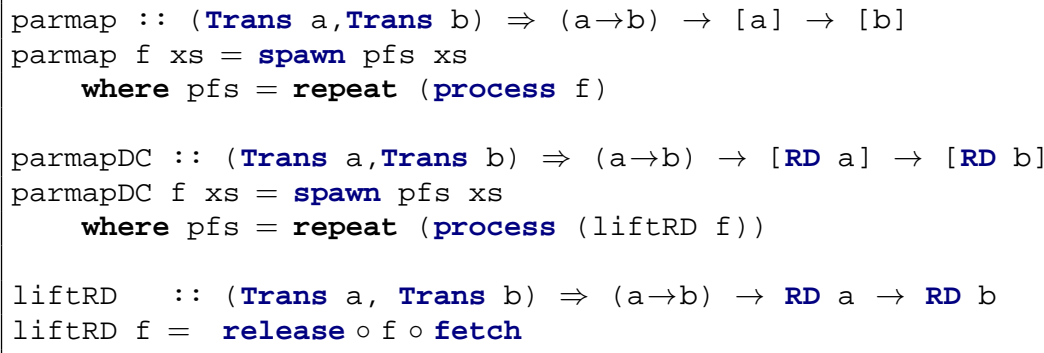

Fig. 4. The parmap and parmapDC skeletons

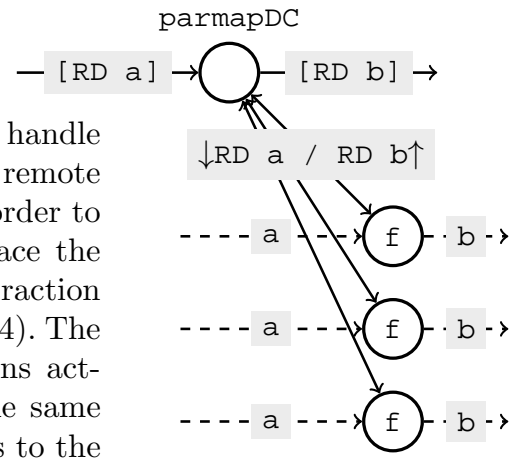

its list element. It can then use this handle to fetch the element directly from the remote place where this element is located. In order to achieve this behaviour, we simply replace the parameter function $\mathrm{f}$ in the process abstraction by its lifted pendant liftRD f (see Fig. 4). The function liftRD is used to lift functions acting on data to functions performing the same computation on remote data. This leads to the skeleton parmapDC where the ending DC stands for Directly Composable due to the remote data interface. This interface makes it possible Fig. 5. Visualization of the parmapDC skeleton for skeletons to receive distributed input and to produce distributed output which is crucial for an efficient composition of skeletons. Fig. 5 visualises the behaviour and communication paths of the parmapDC skeleton. The upper circle represents the process evaluating the parmapDC instantiation. It generates the other processes whose task is to apply the parameter function $f$ to input of type a and produce output of type $b$. Note that only remote data handles for the input and the output values are communicated between the generator process and its child processes. The proper data is communicated via dynamic channel connections indicated by dashed lines.

\subsection{The allToAllDC skeleton}

In Figure 6 we present an all-to-all skeleton allToAl1DC. This skeleton depends inherently on its inner communication pattern which we will implement using remote data. We need the following variants of the remote data interface functions in order to fetch or release a list of remote data:

- releaseAll : : [a] $\rightarrow[\mathrm{RD}$ a] is defined as map release. 
Dieterle, Horstmeyer, Loogen, Skeleton Composition using R. D. In: PADL 2010, LNCS 5937. (C) 2010 Springer Berlin/Heidelberg. The original publication is available at springerlink.com

- fetchAll : : [RD a] $\rightarrow$ [a] is semantically equivalent to map fetch, but needs a special eager implementation which initiates to fetch each input list element without waiting for the result of this action.

The input of the allTOAllDC skeleton is a list of remote data with, say, $n$ elements and two transformation functions $t 1$ and $t 2$ to allow the processes to transform the input data before sending data to all other processes and after
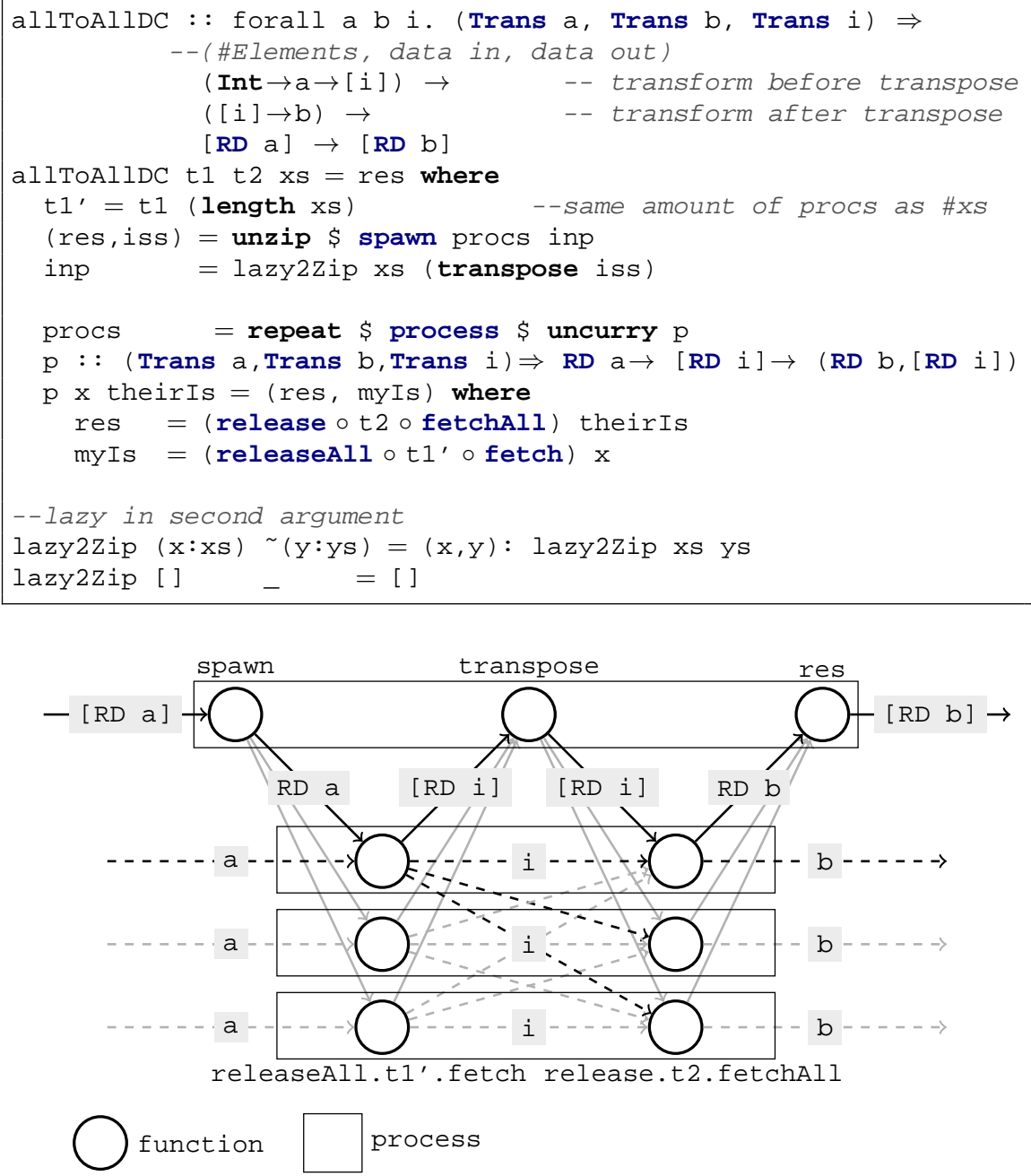

Fig. 6. The allTOAllDC skeleton: code and visualisation. (The darker shading of the arrows from the uppermost child process emphasizes the connectivity of a single process.) 
Dieterle, Horstmeyer, Loogen, Skeleton Composition using R. D. In: PADL 2010, LNCS 5937. (C) 2010 Springer Berlin/Heidelberg. The original publication is available at springerlink.com

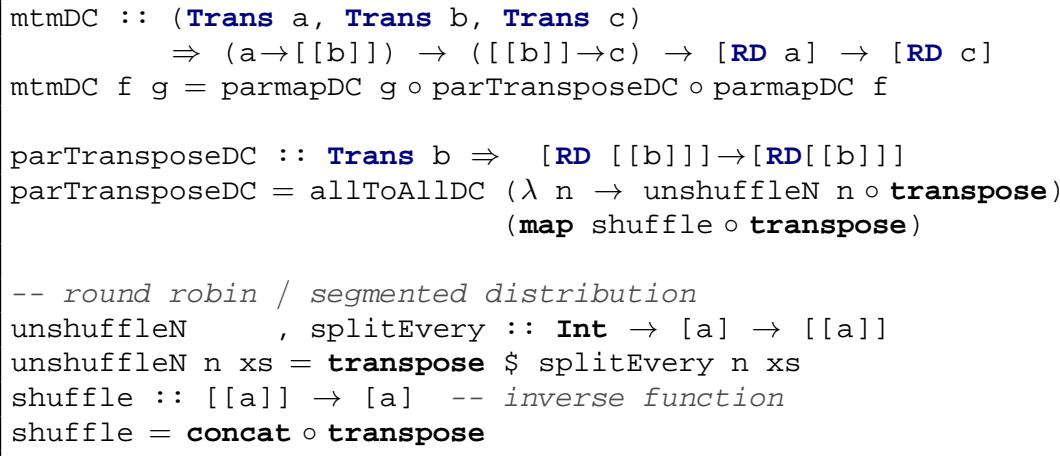

Fig. 7. Composition of parmap and transpose skeletons

receiving data from all other processes, respectively. The length of the input list determines the number of processes to be created by spawn. Every process will fetch its remote input $x$ and transform it with the transformation function $t 1$. This yields a list of intermediate data for each child process which is released element-wise by releaseAll, giving the list my Is : : [RD i] with remote data handles. Note that this list must have the same number $n$ of elements as the input list. This list of remote data handles is returned to the root process in the second component of each process's result tuple. The root process receives one such list from each of its child processes resulting in the $n \times n$ matrix iss : : [ [ $[\mathrm{RD}$ i] ] . It transposes this matrix and sends the result back to the processes as its second, lazily supplied parameter theirIs. Each process gets thus one remote intermediate value of type $\mathrm{RD} i$ of each sibling process and of itself. The values are gathered using fetchAll, transformed by the second parameter function $t 2$ to the output type $\mathrm{b}$ and released. The visualisation in Fig. 6 again shows the exchange of remote data handles between the root process the child processes and using dashed arrow the direct communication of data between the processes.

\subsection{Composing Skeletons with Remote Data Interface}

The allToAllDC skeleton can be used to express arbitrary data exchange that requires an all-to-all network. A common special case is the transposition of a matrix which is distributed over several processes. The way the matrix is distributed over the processes can be manifold. Each process might be assigned e.g. to one row or - more general - to several rows of the matrix. In the example skeleton parTransposeDC of Fig. 7, we implement the more general case. Thus, we are not restricted to 1:1 relations between rows and processes. We assume that rows are distributed round robin over the processes. The advantage against a block distribution is that the matrix can be assigned partially to the processes without knowledge of the overall number of rows. Hence, the transposition skeleton has to assign the columns of the overall matrix (rows of 

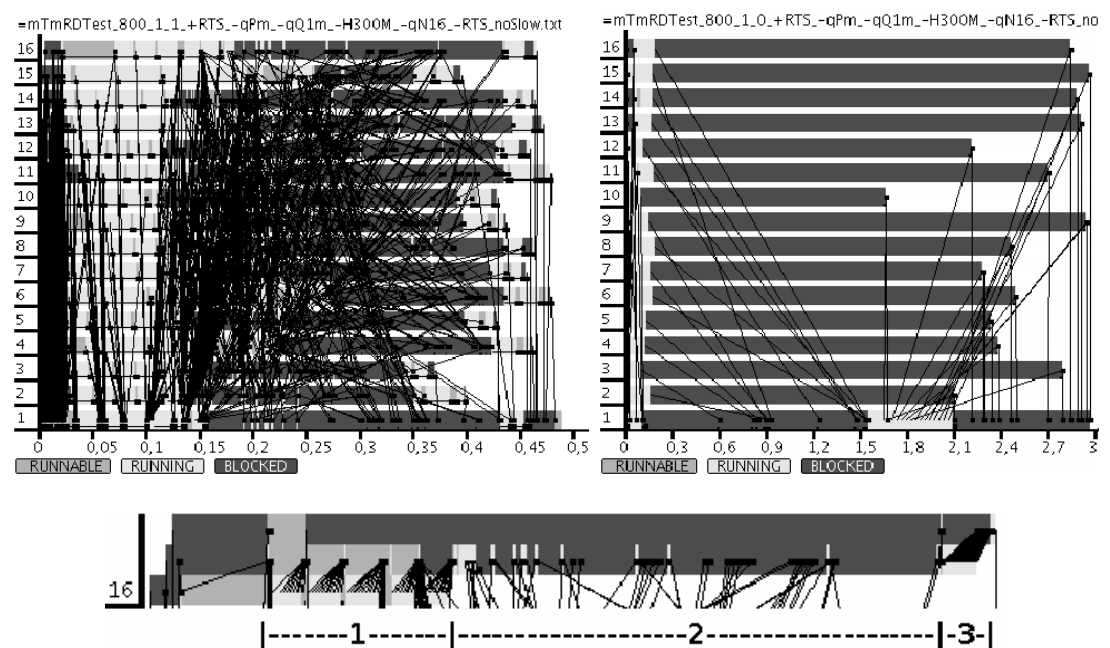

Fig. 8. Runtime behaviour of the skeleton $\mathrm{mtmDC}$ in the global view(left), the zoomed process on Machine 16 (bottom) vs. the local transposition version (right).

(Note the different scaling of the x-axes in the upper traces and that the zoomed view has been taken from a processes-per-machine view, here showing the activity bars of the three processes on Machine 16.)

the transposed matrix) round robin to the processes. The first transformation function of type Int $\rightarrow[[b]] \rightarrow[[[b]]]$ first transposes a list of rows to get the list of the former columns. In a second step, these are round robin distributed to sublists, one for each process. Process $i$ will consequently receive one row-sliced and column-sliced partial matrix from each process. The second transformation of type [ [ [b] ] ] $\rightarrow[[b]]$ will shuffle the row-slices (transposed column-slices) into each other to recover the rows of the overall transposed matrix. This is done by flipping the outer dimension (the list of partial matrices) with the row-dimension using transpose. Thus every outer list element contains all partial rows belonging to the same row of the overall matrix. The transformation map shuffle re-establishes each row.

Now, we can combine the parmapDC skeleton of Fig. 4 and the parallel transpose skeleton parTransposeDC in the function mtmDC (cf. Fig. 7), a parallel version of the function composition map $g \circ$ transpose $\circ$ map $f$. Without remote data a naive parallel implementation would be

$$
\text { parmap } g \circ \text { unshuffleN } n \circ \text { transpose o shuffle o parmap } f
$$

This version gathers the data for the intermediate transposition step in the caller process.

We compared runtime activity profiles of the mtmDC skeleton with the naive version. In our example executions, the parameter functions $f$ and $g$ have been set to the dummy function map (scanll (+)) which creates rows of prefix sums. The input matrix contained the number 1 in each position. 
Dieterle, Horstmeyer, Loogen, Skeleton Composition using R. D. In: PADL 2010, LNCS 5937. (C) 2010 Springer Berlin/Heidelberg. The original publication is available at springerlink.com

In order to focus on communications in the middle part of the composed skeletons, input and output communications have been suppressed in the runtime traces underlying the activity profiles. Moreover, the default streaming mode of the communication has been replaced by a single message mode to reduce the number of messages exchanged between the processes.

Each skeleton was instantiated with an input matrix of size $800 \times 800$ and evaluated on 8 Intel Core 2 Duo machines with a Fast Ethernet connection, where each processor core hosted two virtual machines of the Eden runtime system. In Fig. 8, we present the activity profiles of the corresponding runtime traces for the two skeletons. The trace visualisations show the activity of each machine on a horizontal bar. The different activity phases of the virtual machines (runnable, running, blocked) are indicated by different colours explained in the traces legend. Messages are depicted by black lines with an arrow (black dot) on the receiver side. The $x$-axis shows the time in seconds.

The upper left trace in Fig. 8 clearly reveals the distributed transposition by the multitude of messages exchanged right after the initial data generation phase and the first map-phase, which is depicted "running" in the trace. The exchange of remote data starts very early overlapping the map-phase and forming dense bundles of messages. The second map-phase at the end of the program execution is rather short. Note that the overall runtime was less than 0.5 seconds.

We have placed the $i$ th process of every skeleton on the same machine, such that communication costs are low. The lower zoomed view of the figure shows the activity bars of the three processes located on the virtual machine 16 . The lowest bar belongs to a child of the first parmapDC-instantiation. The upper two bars show the processes of the parallel transpose skeleton and the second parmap instantiation. With this information, we can easily identify the different types of messages. During phase 1 the process of the first parmapDC skeleton sends its results to the parTransposeDC process. In the second phase the intermediate data is exchanged with the processes on the other machines. Finally, in phase 3, the result of the transposition is passed on to the second parmapDC process.

The upper right trace in Fig. 8 belongs to the naive version which performs a local transposition in the root process. As expected, this version is much slower with an overall runtime of approximately 3 seconds. The conspicuously fast communication between machine 1 and machine 10 is because the two virtual machines share the same physical machine. Further tests with varying input sizes (not shown) confirmed the enormous runtime advantages of the distributed version.

\subsection{The allReduceDC skeleton}

The all-reduce skeleton combines distributed data using a binary reduction function. It leaves the result duplicated on all processes involved in the reduction. Usually, it is implemented using the classical butterfly scheme which is also a common way to efficiently synchronise data between parallel processes. As for the allToAlIDC skeleton, it is crucial for the all-reduce skeleton that data is 
Dieterle, Horstmeyer, Loogen, Skeleton Composition using R. D. In: PADL 2010, LNCS 5937. (C) 2010 Springer Berlin/Heidelberg. The original publication is available at springerlink.com

transferred to and from the skeleton in a distributed way. The butterfly reduction for $n$ processes is done in logn parallel communication and local reduction steps. In each step, the communication partner of process $k$ is usually calculated with the boolean function $k$ xor $2^{\text {step-1 }}$.

Fig. 9 shows the definition of the function bitFlipF which applies a transformational way to determine the communication partner for the current step. The input list xs contains at position $j$ the value of process $j$. xs is distributed round robin to $d=\left(2^{\wedge} \mathrm{step}\right)$ sublists. The values to be exchanged are in the same columns of the transformed matrix. Their indexes differ by $2^{\text {step-1 }}$ which equals d 'div' 2 or half the number of inner lists. We flip the first half of inner lists with the second half and achieve the desired value exchange. A function call to shuffle re-establishes the original list structure.

The allReduceDC (see Figure 10) skeleton uses the function bitFlipF to rearrange lists of remote data in the caller process which represent the results of the intermediate reduction steps of the skeleton's processes. The rearranged lists are sent back to the processes. Thus, each process gets the remote values released by one partner in every step. Fetching these values establishes the butterfly communication topology.

The skeleton's input is a list with $2^{\text {steps }}$ remote data handles ${ }^{1}$. For each handle a process will be instantiated. The skeleton takes two parameter functions: function initF : : $a \rightarrow b$ is used to transform the initial remote value of each process after it is fetched. This transformation allows to work with different types for the input values and the reduction function inputs. The reduction function redF $:: \mathrm{b} \rightarrow \mathrm{b} \rightarrow \mathrm{b}$ which should be associative and commutative is applied in each step to the results of the previous step of a process and of its partner. This behaviour can concisely be expressed with scanl1 redF applied to the stream toReduce of values to be reduced. The stream toReduce is composed of the initial value and the stream input theirReds. The latter contains the partners' values for all steps. Note that the complete list structure of theirReds is already built in theirReds' even before its first element is received. Thus the request for all remote values can be eagerly initiated by the function fetchAll which would otherwise block on an incomplete list structure. The result of the scanl1 application is element-wise released in every process, resulting in a list of remote data which is also generated in advance. This happens because the evaluation of releaseAll equally depends only on its parameter list's structure.

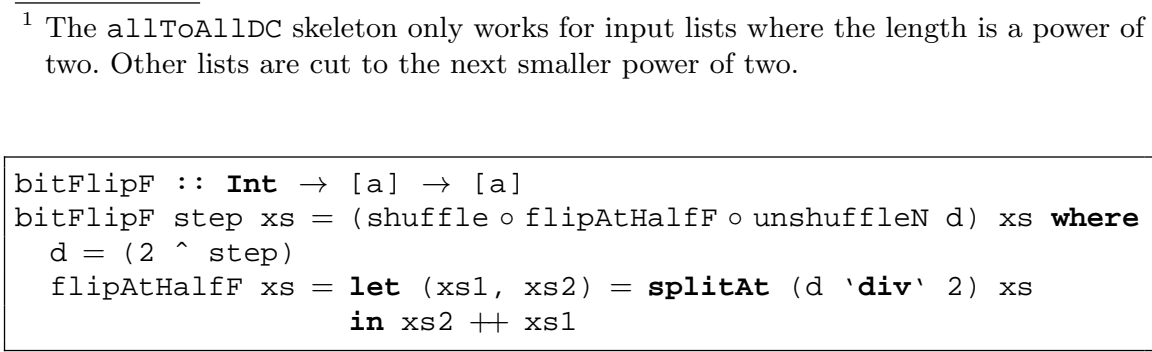

Fig. 9. Flip of values at bit $1 \mathrm{di}$ 
Dieterle, Horstmeyer, Loogen, Skeleton Composition using R. D. In: PADL 2010, LNCS 5937. (C) 2010 Springer Berlin/Heidelberg. The original publication is available at springerlink.com

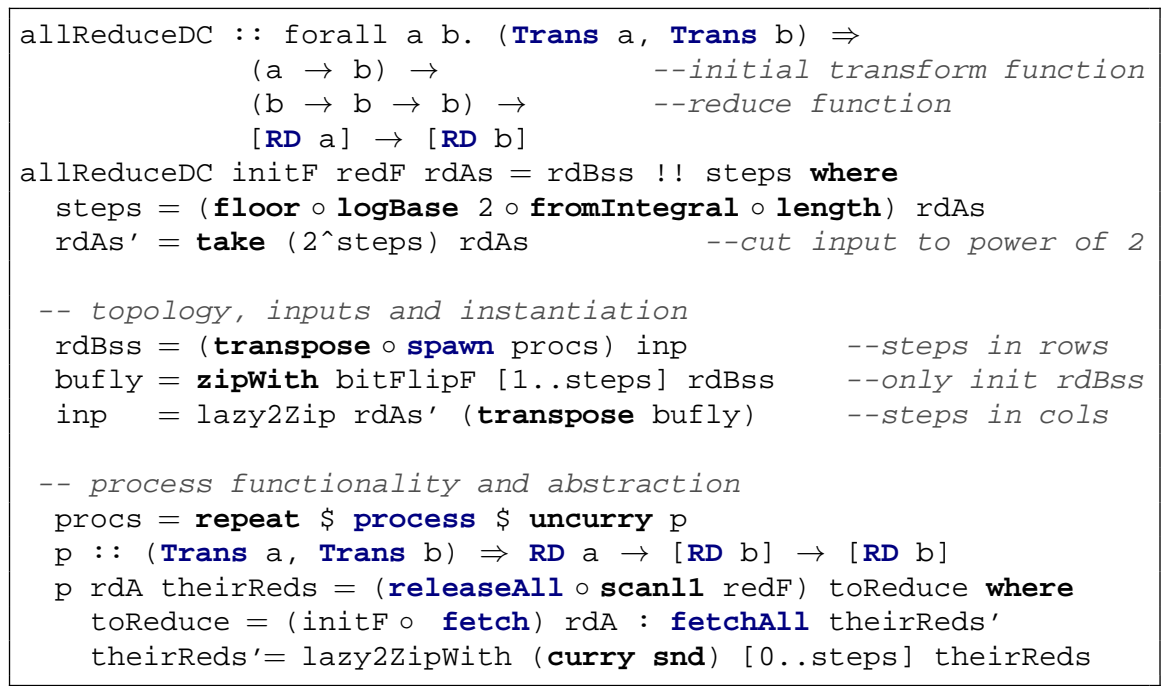

Fig. 10. The allReduceDC skeleton

Thus the exchange of remote data handles via the root process can happen in advance, independently of the parallel reduction steps.

The caller process gathers the result streams of all processes in a nested list. We transpose this list to have all remote values of a step in each inner list of rdBss. Applying the function bitflipF to the first steps lists permutes these according to the butterfly scheme. We transpose this permutation bufly such that each process's input is located in one inner list. This transposed list is lazily zipped with the initially supplied input list rdAs using lazy2zip and passed back to the processes. The final result consists of the results of the last reduction step, i.e. the last element of the list rdBss.

We have tested the allReduceDC skeleton with a dummy example which we executed on an 8 core Intel Xeon machine. The initial transformation function initF serves as generator and generates the list [1.nElems], where nElems is a parameter of the program and in our example set to 200000. The trace visualisation in Fig. 11 reveals interchanging computation and communication phases. The butterfly interconnection scheme can clearly be recognised in the messages exchanged between the processes. The generation of elements is depicted as the first "running" phase. The reduction network has been set up before, by exchanging the remote data messages via the root process on Machine 1 (initial messages). Three reduction phases follow. First the direct neighbours exchange their lists leading to the typical butterfly pattern of messages. The processes reduce their lists using the reduction function redF which is set to zipwith $(+)$. For the next steps, the distance to the partner process is doubled every time. Finally, a parmapDC skeleton is called to consume the data and return an empty list to the root process. 


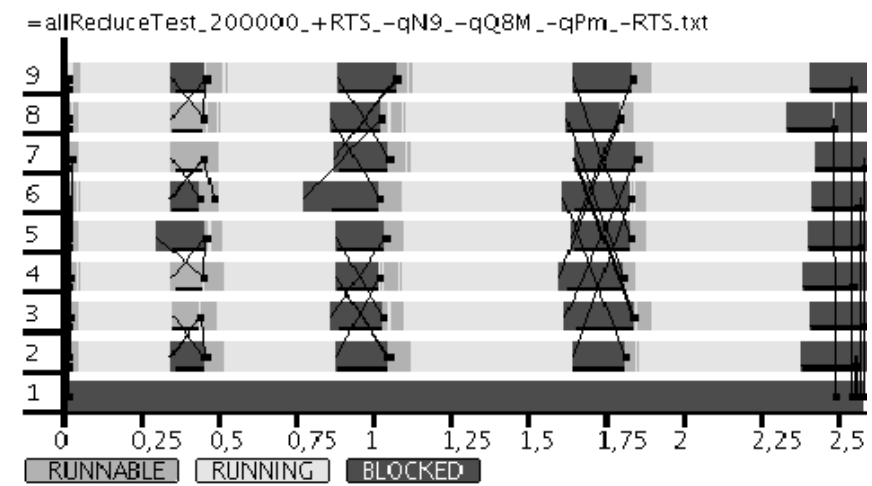

Fig. 11. Runtime behaviour of the allReduceDC skeleton

\section{Related Work}

Alt and Gorlatch [1-3] introduced a concept similar to remote data called remote references in the context of optimisations of Java RMI. They concentrated on what they called lazy RMI, localised RMI and future-based RMI. Lazy RMI describes the basic functionality. Future-based RMI allows to create and pass remote references before the corresponding values are computed. We get this in Eden for free because of Haskell's laziness. An optimization for data passed locally on the same machine, like localised RMI, would be a good optimisation of the Eden runtime system, but is currently not implemented.

Alternative approaches to skeleton composition are based on the use of distributed data structures. Kuchen and Cole [7] describe a skeleton library based on $\mathrm{C}++$ and MPI which integrates task and data parallel skeletons. Darlington [6] uses an imperative base as well but describes the composition of (predefined) skeletons itself functionally in the structured coordination language (SCL).

Although programming with distributed data structures is comfortable and efficient, the number of predifined data structures is limited and their use is thus not as flexible as working with remote data. Remote data can be nested in arbitrary algebraic data structures and manipulated by standard functions on those structures.

\section{Conclusions and Future Work}

The remote data concept uses an existing communication topology to build direct connections between different processes. Existing bottlenecks are thereby circumvented and the total communication amount is reduced. Although being language-independent the concept enrolls its power and expressive elegance especially in the context of a declarative host language like Eden, where the concept 
Dieterle, Horstmeyer, Loogen, Skeleton Composition using R. D. In: PADL 2010, LNCS 5937. (C) 2010 Springer Berlin/Heidelberg. The original publication is available at springerlink.com

itself is implemented with small effort and only minor changes to existing code are needed to lift functional results to the new data type.

Algorithmic skeletons that define process networks with complex communication patterns can be defined in an elegant and concise way. Composition of skeletons with a remote data interface enables direct communication between processes within the different skeleton instances. Communication overhead is substantially reduced and skeleton compositions do not suffer anymore from the performance penalty caused by the collection and redistribution of distributed data in ordinary settings. Thus, the remote data concept enhances modularity of skeleton-based parallel programming, especially by promoting easily composable skeletons.

With remote data the explicit channel handling using new and parfill can in most cases be abandoned. This improves the the elegance and usability of Eden even more. We think that there is room for improvements in other parts of the language as well. One of the topics that has been brought up many times is the question whether the Eden functions should get an IO-interface or remain unchanged. We plan an intensive study of the benefits and drawbacks of an language specification that makes the side effects explicit.

\section{References}

1. M. Alt. Using Algorithmic Skeletons for Efficient Grid Computing with Predictable Performance. PhD thesis, Universität Münster, July 2007.

2. M. Alt and S. Gorlatch. Future-Based RMI: Optimizing compositions of remote method calls on the Grid. In H. Kosch, L. Böszörményi, and H. Hellwagner, editors, Euro-Par 2003, volume 2790 of Lecture Notes in Computer Science, pages 682-693. Springer-Verlag, Aug. 2003.

3. M. Alt and S. Gorlatch. Adapting Java RMI for grid computing. Future Generation Computer Systems, 21(5):699-707, 2004.

4. J. Berthold, M. Dieterle, and R. Loogen. Implementing parallel google map-reduce in eden. In H. Sips, D. Epema, and H. Lin, editors, Euro-Par 2009, volume 5704 of Lecture Notes in Computer Science, pages 990-1002. Springer-Verlag, 2009.

5. M. Cole. Algorithmic Skeletons: Structured Management of Parallel Computation. MIT Press, 1989.

6. J. Darlington, Y.-k. Guo, H. W. To, and J. Yang. Parallel skeletons for structured composition. In PPOPP '95: Proceedings of the fifth ACM SIGPLAN symposium on Principles and practice of parallel programming, pages 19-28, New York, NY, USA, 1995. ACM.

7. H. Kuchen and M. Cole. The integration of task and data parallel skeletons. Parallel Processing Letters, 12(2):141-155, 2002.

8. O. Lobachev, J. Berthold, M. Dieterle, and R. Loogen. Parallel fft using eden skeletons. In 10th Intl. Conference on Parallel Computing Technologies (PaCT)'09, volume 5698 of Lecture Notes in Computer Science. Springer-Verlag, 2009.

9. R. Loogen, Y. Ortega-Mallén, and R. Peña-Marí. Parallel Functional Programming in Eden. Journal of Functional Programming, 15(3):431-475, 2005. 